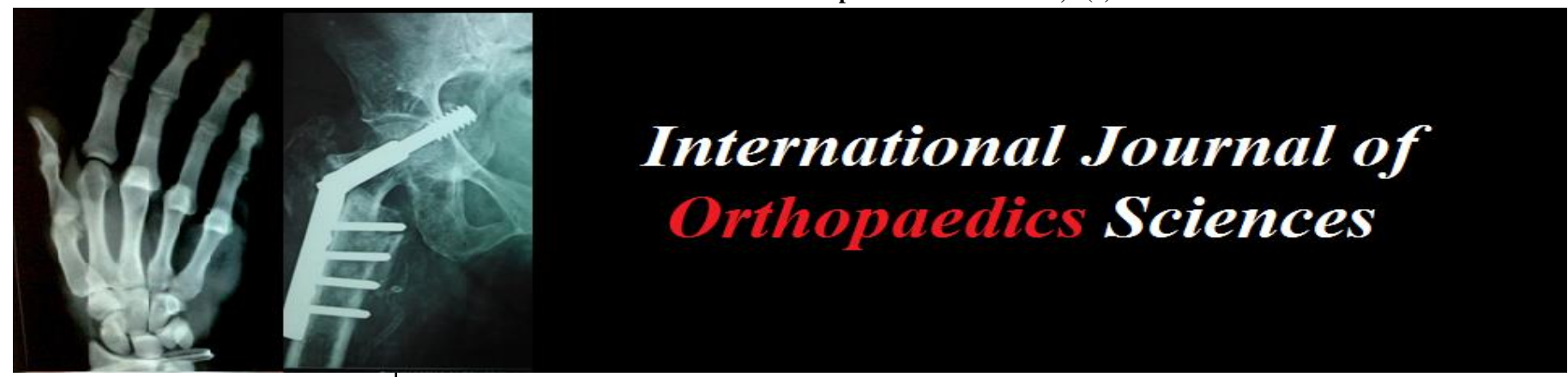

E-ISSN: 2395-1958

P-ISSN: 2706-6630

IJOS 2020; 6(1): 183-185

(C) 2020 IJOS

www.orthopaper.com

Received: 06-11-2019

Accepted: 10-12-2019

Dr. Hemeshwar Harshwardhan Professor \& Unit Head,

Department of Orthopaedics.

JLN Medical College \& Hospital,

Ajmer, Rajasthan, India

Dr. Gaurav Kumar Laddha

Post Graduate Trainee,

Department of Orthopaedics,

JLN Medical College \& Hospital,

Ajmer, Rajasthan, India

Dr. Prashant Gupta

Post Graduate Trainee,

Department of Orthopaedics,

JLN Medical College\& Hospital,

Ajmer, Rajasthan, India
Corresponding Author:

Dr. Gaurav Kumar Laddha

Post Graduate Trainee,

Department of Orthopaedics,

JLN Medical College \& Hospital,

Ajmer, Rajasthan, India

\section{Outcome assesment of proximal fibular osteotomy in medial compartment knee osteoarthritis}

\author{
Dr. Hemeshwar Harshwardhan, Dr. Gaurav Kumar Laddha and Dr. \\ Prashant Gupta
}

DOI: https://doi.org/10.22271/ortho.2020.v6.i1d.1858

\section{Abstract}

Objective: The aim of the study was outcome assessment of proximal fibular osteotomy in medial compartment knee osteoarthritis.

Method: This study was conducted in Dept. of Orthopedic J.L.N. medical collage Ajmer. Thirty five patients in between 40-71 years age group were taken in this study. Patient with moderate to severe symptomatic medial compartment $\mathrm{OA}$ of the knee and whom conservative management has failed were included in this study. Assessment of knee pain was done using a visual analogue scale and knee functional activities were evaluated using the WOMAC score. Preoperative and postoperative weightbearing and whole lower extremity radiographs were obtained to analyse the alignment of the lower extremity by tibio-femoral angle and mechanical axis deviation.

Results: VAS score and WOMAC score for pain relief and functional activities was significantly improved. Weight-bearing lower extremity radiographs showed significant change in tibio-femoral angle and mechanical axis.

Conclusion: Proximal fibular osteotomy may reduce knee pain significantly in the varus osteoarthritic knee and improve the functional recovery of the knee joint.

Keywords: Knee osteoarthritis, proximal fibular osteotomy, VAS score, WOMAC score

\section{Introduction}

Knee osteoarthritis (OA) is a chronic, progressive degenerative disease with accompanying joint pain, stiffness, and deformity ${ }^{[1]}$. It is the most common cause of disability in the older population. Disability is caused by pain and limitations in mobility. High tibial osteotomy, unicompartment knee arthroplasty and total knee arthroplasty are the methods used for treating knee OA. High tibial osteotomy is a technically demanding procedure and may result in complications, including neurovascular injury, iatrogenic fracture, and nonunion [2, 3]. Total knee arthroplasty can correct lower extremity alignment, relieve pain, and improve function significantly. However, for younger, active patients or patients with moderate OA, it may not be the treatment of choice ${ }^{[4]}$.

Proximal fibular osteotomy (PFO) is an alternative treatment to high tibial osteotomy (HTO). It is a surgical procedure for medial compartment knee osteoarthritis (KOA). Compared to HTO, PFO has several advantages. PFO is a comparatively simple surgical procedure compared to HTO. PFO is less invasive with a very short incision, requires limited tissue dissection and no internal fixation is implanted. The postoperative recovery period is faster than with HTO. PFO is associated with few complications. Similar to HTO, PFO can relieve the symptoms of KOA with realignment of the lower extremity.

\section{Materials and Methods}

This study was conducted in Department of Orthopaedics, JLN Medical College \& Associated Group of Hospitals, Ajmer from January 2018 to October 2019. Thirty Patients with medial compartment osteoarthritis of knee were taken in the study. Patients with moderate to severe symptomatic medial compartment $\mathrm{OA}$ of the knee, in whom conservative management has failed and who have radiographic evidence of significant varus were taken in the study. Patients with bicompartmental or tricompartmental osteoarthritis knee and ligamentous 
instability were not included in this study.

Operative procedure was performed under spinal anesthesia. We routinely place a tourniquet on the thigh and drape only the knee free. A small buttress is mounted to the operating table to support the heel in $90^{\circ}$ flexion of the knee. Perioperative intravenous antibiotic prophylaxis was used. First, the fibular head was marked. To avoid injury to the common peroneal nerve and tibial attachments of the soft tissue structures crossing the knee joint. A lateral incision of 3 to $5 \mathrm{~cm}$ was made at the proximal third of the fibula. The fascia was then incised in line with the septum between the peroneus and soleus, the muscles were separated, and the fibula was exposed. A $2 \mathrm{~cm}$ section of the fibula was removed 6 to $10 \mathrm{~cm}$ below the fibular head with the use of an oscillating saw or multiple drill holes followed by osteotome. Following resection, the fibula ends were sealed with bone wax. The muscles, fascia, and skin were sutured separately after the incision had been irrigated with a large volume of normal saline.

In this study Western Ontario \& McMaster Universities Index (WOMAC) score and Visual Analogue Scale (VAS) were used to assess functional results. Pain being single most important factor of functional outcome, was assessed using the Visual analogue scale. WOMAC score was used to analyse the pain, stiffness and functional activity.

Preoperative and postoperative weight-bearing and whole lower extremity radiographs were obtained to know the Mechanical axis deviation and Femorotibial Angle to analyse the alignment of the lower extremity.

\section{Results}

A total of 35 patients were included in this study. In this study average age of patients was 55.34 years \pm 8.47 per knee with a range of 40 to 71 years. In this study predominantly $57.14 \%$ were females while $42.85 \%$ were male.

The mean preoperatively value of VAS score was 7.50 with standard deviation 0.56 and mean postoperative value of VAS score was 1.60 with standard deviation 1.10 and $\mathrm{p}$ value was significant (Figure-1).

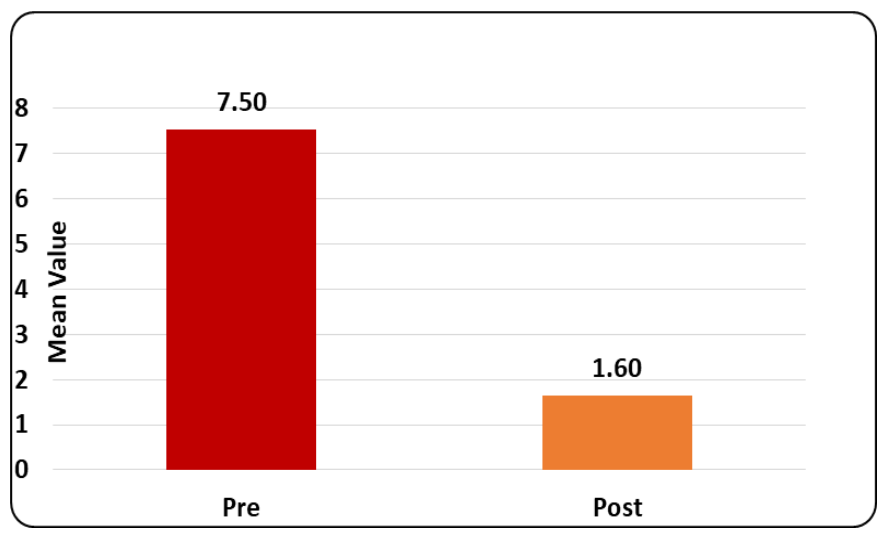

Fig 1: VAS score

The mean preoperatively value of womac score was 48.2 with standard deviation 3.69 and mean postoperative value of womac score was 22.2 with standard deviation 3.90 and $\mathrm{p}$ value was Significant (Figure-2).

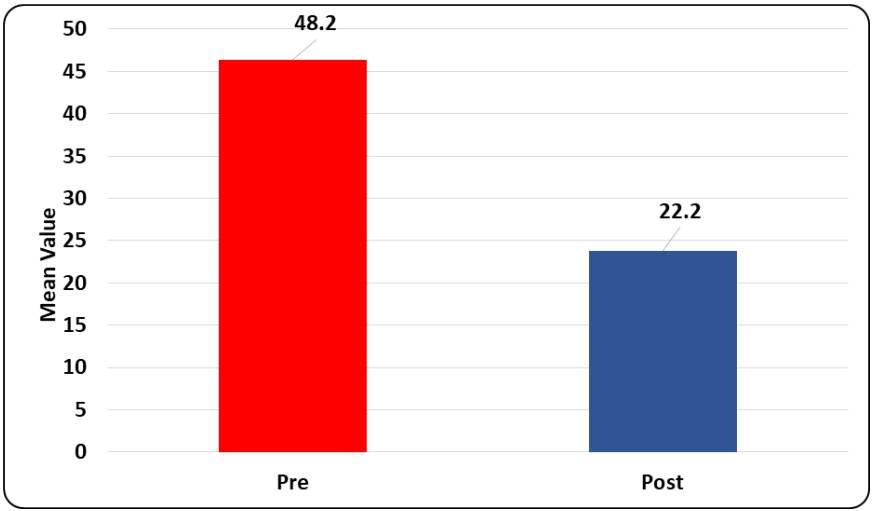

Fig 2: Womac score

The mean preoperatively value of Mechanical Axis Deviation was $11.14 \mathrm{~mm}$ with standard deviation 3.75 and mean postoperative value of Mechanical Axis Deviation was $9.34 \mathrm{~mm}$ with standard deviation 3.53 and $\mathrm{p}$ value was significant (Figure-3).

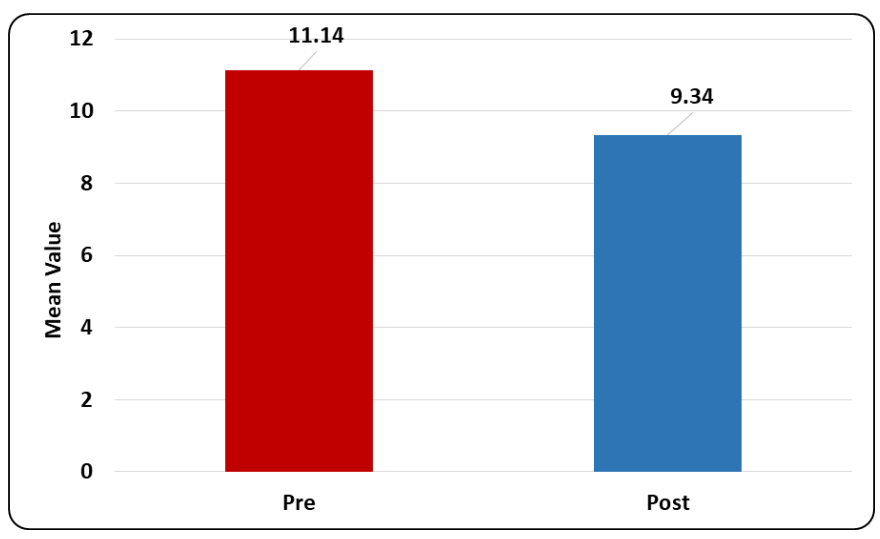

Fig 3: Mechanical axis deviation

The mean preoperatively value of Femorotibial angle was 182.6 with standard deviation 1.47 and mean postoperative value of Femorotibial angle was 174.03 with standard deviation 1.75 and p value was significant. (Figure-4).

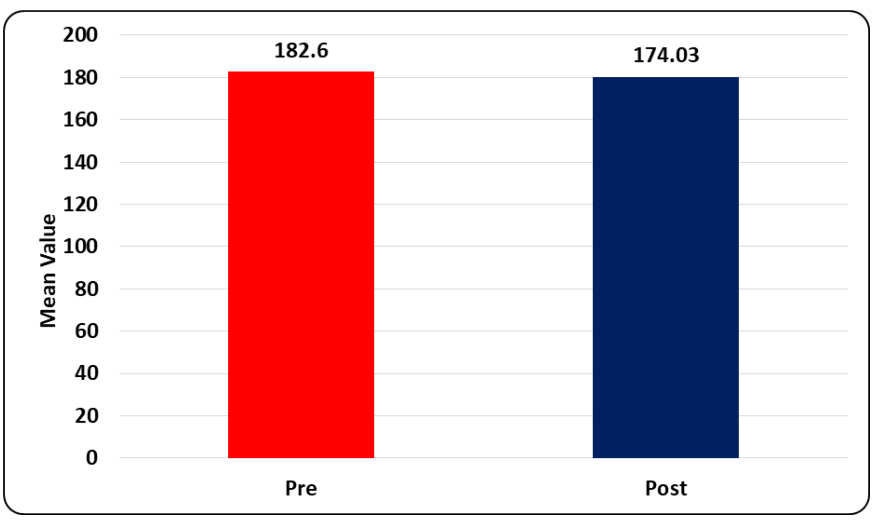

Fig 4: Femorotibial angle

The most frequent complication in this series was superficial peroneal nerve palsy. A total of 4 patients got superficial peroneal nerve palsy of the 35 that were applied amounting to $11.42 \%$. All of these complications were completely recover within 6 months. 


\section{Discussion}

Proximal fibular osteotomy is the surgical method of choice in certain specific indications for knees with medial compartmental osteoarthritis. The major advantage of the operation is that it allows unlimited activity to the patient. Thus, for patients who have an occupation requiring vigorous activity or who wish to continue playing sports, an osteotomy is a reasonable procedure that in no way precludes a later total knee arthroplasty.

In our study mean preoperative VAS score was $7.50 \pm 0.56$ and postoperative VAS score was decrease to $1.60 \pm 1.10$ which was statistically significant. Xiaohu Wang et al. ${ }^{[5]}$ and Guoping Zou et al. ${ }^{[6]}$ (2017) also showed the mean visual analogue scale scores significantly decreased in their study.

Preoperative mean womac score was $48.2 \pm 3.69$ which was significant decrease to $22.2 \pm 3.90$ postoperatively.

Regarding the measure of correction two measures were taken: Mechanical axis deviation and Femorotibial Angle. Femorotibial angle is easiest measure and follow but least precise due normal variations of length of femur, tibia and femoral neck shaft angle in different populations. Mechanical axis deviation on tibial plateau on the other hand is most precise and doesn't get affected variations in population.

In our study, the mean FTA was $182.6^{\circ} \pm 1.47$ preoperatively and after correction postoperatively mean angle was decrease to $174.03^{\circ} \pm 1.75$ which was statistically significant. The mean femorotibial angle was decrease which was also satistically significant in the study done by Zong-You Yang et al. ${ }^{[7]}$ and by Guoping Zou et al.

In our study, the mean mechanical axis deviation was $11.14 \pm$ $3.75 \mathrm{~mm}$ preoperatively and postoperatively mean mechanical axis deviation was significantly decreased.

The most frequent complication in this study was superficial peroneal nerve palsy. In the study done by Zong-You Yang et al. 81 also showed this complication.

\section{Conclusion}

Proximal fibular osteotomy may reduce knee pain significantly in the varus osteoarthritic knee and improve the functional recovery of the knee joint. It is a safe, simple, and effective procedure that is an alternative to HTO and may delay or even negate the need for total knee arthroplasty for medial compartment OA of the knee joint. Care must be taken to avoid potential nerve injuries.

\section{References}

1. Focht BC. Move to improve: how knee osteoarthritis patients can use exercise to enhance quality of life. ACSM's Health Fit J. 2012; 16:24-28.

2. Wu LD, Hahne HJ, Hassenpflug T. A long-term followup study of high tibial osteotomy for medial compartment osteoarthrosis. Chin J Traumatol. 2004; 7:348-353.

3. Sprenger TR, Doerzbacher JF. Tibial osteotרomy for the treatment of varus gonarthrosis: survival and failure analysis to twenty-two years. J Bone Joint Surg Am. 2003; 85:469-474.

4. Schnurr C, Jarrous M, Gudden I, Eysel P, Konig DP. Preoperative arthritis severity as a predictor for total knee arthroplasty Patients satisfaction. Int Orthop. 2013; 37(7):1257-1261.

5. Xiaohu Wang, Lei Wei. Proximal fibular osteotomy: a new surgery for pain relief and improvement of joint function in patients with knee osteoarthritis; Journal of International Medical Research. 2017; 45(1):282-289.

6. Guoping Zou, Weibin Lan. Early clinical effect of proximal fibular osteotomy on knee osteoarthritis. Biomedical Research. 2017; 28(21):9291-929.

7. Yang ZY, Chen W, Li CX et al. Medial compartment decompression by fibular osteotomy to treat medial compartment knee osteoarthritis: a pilot study. Orthopedics. 2015; 38:e1110-e1114. 